\title{
CARACTERIZAÇÃO PRELIMINAR DE SEDIMENTOS DE CAVERNAS EM SISTEMAS CÁRSTICOS DO PRIMEIRO PLANALTO PARANAENSE ${ }^{1}$
}

\author{
PRELIMINARY CHARACTERIZATION OF CAVE SEDIMENTS IN KARSTIC \\ SYSTEMS IN THE FIRST PLATEAU OF THE STATE OF PARANA, BRAZIL
}

\author{
Angelo Evaristo SIRTOLI2 \\ Marcelo Ricardo de LIMA ${ }^{3}$
}

\begin{abstract}
RESUMO
Solos em cavernas são formados em sua maior parte de sedimentos provenientes do exterior através dos rios subterrâneos e precipitação de substâncias solúveis na água, acrescidos de material orgânico dos animais e microorganismos que habitam este ecossistema. Este trabalho visou avaliar características químicas e físicas de sedimentos de cavernas, em diferentes sistemas cársticos. Foram estudadas as cavernas principais dos sistemas de Bromado e Cachimba (Rio Branco do Sul - PR) e Pinheirinho (Campo Largo $\mathrm{PR}$ ). Foram realizadas análises químicas e granulométricas dos sedimentos. $\mathrm{O} \mathrm{pH}$ destes, na maioria dos casos, é alcalino ou ligeiramente ácido. Os maiores teores de $\mathrm{C}$ orgânico situam-se nas camadas superficiais, nos níveis de base atuais e pretéritos. Os teores de $\mathrm{P}$ disponível são altos, porém pode ter ocorrido extração de formas pouco lábeis em decorrência do extrator utilizado. Os teores de $\mathrm{K}$ são médios a baixos e, a CTC e saturação de bases são altas. Os sedimentos apresentam textura média e argilosa, ressaltando que as amostras com maiores concentrações de areia grossa são aquelas no nível de base atual, podendo indicar a degradação do entorno das cavernas. Os resultados encontrados evidenciam a possibilidade de utilização destas informações para subsidiar o estudo dos impactos antrópicos nas cavernas.
\end{abstract}

Palavras-chave: solo, calcário, sedimento, espeleologia.

\begin{abstract}
The soil in caves is formed largely by eroded sediments carried from the outside by rivers and precipitation of soluble substances. Organic materials from the surrounding ecosystems are also added to the sediments in caves. Agricultural and forestry activities in karstic areas may cause impacts in these caves. The purpose of this work was to evaluate chemical and granulometric characteristics of cave sediments, in three different karstic systems. The studied caves were the karstic systems of Bromado and Cachimba (Rio Branco do Sul - PR) and Pinheirinho (Campo Largo - PR). In most cases the pH of the sediments, are alkaline or slightly acidic. Higest levels of organic carbon are found in surface layers, in both current base levels and past base levels. Levels of available $\mathrm{P}$ are high, althought labile $\mathrm{P}$ fraction may also have been extracted. The $\mathrm{K}$ levels are medium to low. CEC and base saturation are high. The sediments are clayey and loamy. The highest concentrations of coarse sand are found in samples from the current base level, which may indicate degradation in areas surrounding the caves. These results may be used as indicators for assessing the human impacts in caves.
\end{abstract}

Key-words: sediments, soil, limestone, speleology.

\footnotetext{
${ }^{1}$ Trabalho apresentado na XXIII Reunião Brasileira de Fertilidade do Solo e Nutrição de Plantas, de 11 a 16 de outubro de 1998, em Caxambu (MG). ${ }^{2}$ Eng. Agr. M.Sc. (CREA 26041D-PR), Professor do Departamento de Solos e Engenharia Agrícola da Universidade Federal do Paraná, Rua dos Funcionários, 1540, 80035-050, Curitiba, Paraná, asirtoli@agrarias.ufpr.br. ${ }^{3}$ Eng. Agr. (CREA 13446V-PR), Doutorando em Agronomia (PGAPVUFPR), Professor do Departamento de Solos e Engenharia Agrícola da UFPR.
} 
SIRTOLI, A.E. e LIMA, M.R. Caracterização preliminar de sedimentos...

\section{INTRODUÇÃO}

Em cavernas o "solo" é formado em certos casos, pela decomposição da rocha matriz "in situ". Porém, na sua maioria são sedimentos (minerais e orgânicos) transportados do meio externo através de rios subterrâneos (sedimentos clásticos), e precipitação de substâncias solúveis na água (sedimentos químicos), que se acumulam em alguns trechos ao longo do percurso do rio e sofrem o acréscimo de material orgânico trazido pelos animais que habitam a caverna. A este material dá-se a denominação de sedimentos de caverna.

Atividades agropecuárias e florestais podem causar impacto em cavernas calcárias (07). Na região metropolitana de Curitiba, além desses impactos, a visitação sem controle, a exploração mineral e a degradação do entorno tem se constituído em ameaça às cavernas, havendo inclusive muitas que foram destruídas sem qualquer registro (13), o que ressalta a importância de estudar as cavernas ainda existentes. Informações sobre os sedimentos destas cavernas podem auxiliar na avaliação de impactos antrópicos recentes nestes ambientes.

Este trabalho teve como objetivo fazer uma caracterização preliminar destes sedimentos de caverna em diferentes sistemas cársticos.

\section{MATERIAL E MÉTODOS}

A área de estudo situa-se no norte da Região Metropolitana de Curitiba, Estado do Paraná, onde encontram-se rochas carbonáticas, pertencentes ao Grupo Setuva e ao Grupo Açungui. Foram estudados os sistemas cársticos das grutas de Bromados (25804'33"S e 49822'48"O) e Cachimba (25809'11"S e 49819'16"O), ambas no Município de Rio Branco do Sul, e da gruta de Pinheirinho (25800'16"S e 49838'07"O), no Município de Campo Largo.

No interior das cavernas, procedeu-se a coleta de sedimentos em diferentes profundidades, amostrando-se três a cinco sítios representativos, dependendo da diversidade de cada caverna. No sistema Cachimba, o sítio 1 localizava-se próximo à ressurgência, na galeria inferior, perto do leito do rio. O sítio 2 estava cerca de $15 \mathrm{~m}$ da entrada da caverna, na galeria superior no antigo leito do rio, com presença de raízes. Os sedimentos desse sítio são provavelmente oriundos de transporte através da dolina que está próxima ou de material de aluvião do antigo leito do rio. O sítio 3 corresponde ao antigo leito do rio, na porção média da caverna. O sítio 4 situava-se no nível de base atual da caverna. O sítio 5 corresponde a um espeleotema decomposto encontrado na parede da caverna. Com exceção do solo do sítio 5 , todos os outros são formados por sedimentos trazidos para a caverna em diferentes épocas, por distintas formas de transporte.
No sistema de Bromados, a amostra do sítio 6 foi coletada na parede da caverna, aproximadamente $3 \mathrm{~m}$ acima do nível das galerias superiores. Este sedimento pode ser oriundo da desagregação de uma lâmina de filito ladeado por calcário ou da desagregação de um espeleotema ("solo de travertino"). O sítio 7 corresponde a um depósito sedimentar na galeria principal, com conexão da dolina que capta águas superficiais. O sítio 8 localizava-se no primeiro nível da caverna (antigo nível de base), onde observou-se grande quantidade de seixos rolados no perfil do solo. O sítio 9 refere-se a coleta efetuada entre escorrimentos calcíticos, tipo cascata de pedra quebrada. Este sítio apresentava-se sob uma fina película de calcita, sendo provavelmente endógeno ou depósito sedimentar muito antigo.

No sistema de Pinheirinho, a amostra do sítio 10 foi coletada na parte final do local conhecido como Salão dos Grube, apresentando material desagregado da parede e de espeleotemas, além de uma mistura de guano. O sítio 11 era no leito de um antigo rio sobre um banco de sedimentos de aproximadamente 10 a $12 \mathrm{~m}$, no local denominado Fonte dos Grube. O sítio 12 situava-se no nível de base atual da caverna.

As amostras de solos e sedimentos foram analisadas quanto a granulometria da fração terra fina (05). Os valores de $\mathrm{pH}$ em $\mathrm{CaCl}_{2} 0,01 \mathrm{~mol} \mathrm{dm}^{-3}$ e os teores de cátions trocáveis $\left(\mathrm{Ca}^{+2}, \mathrm{Mg}^{+2}\right.$ e $\mathrm{Al}^{+3}$ extraídos por $\mathrm{KCl} 1 \mathrm{~mol} \mathrm{dm}^{-3}$, e $\mathrm{K}^{+}$extraído por Mehlich-1), $\mathrm{H}+\mathrm{Al}$ (determinados por correlação com o índice SMP), P disponível (extraído por Mehlich-1) (09) e carbono orgânico (determinado por colorimetria, após oxidação da matéria orgânica por solução de dicromato de sódio em ácido sulfúrico a frio) (11). Também foram calculadas a capacidade de troca de cátions (CTC) e a porcentagem de saturação por bases (V) (05).

\section{RESULTADOS E DISCUSSÃO}

Analisando-se os valores de $\mathrm{pH}$ (Tabela 01) pode-se observar uma pequena amplitude. De maneira geral, os valores de $\mathrm{pH}$ encontrados nos sedimentos são pouco ácidos a subalcalinos, o que concorda com a afirmação de Costa (04) que os calcários podem originar solos neutro a subalcalinos. Esses valores de $\mathrm{pH}$ se equiparam aos resultados de $\mathrm{pH}$ em água encontrados por Basso (02) na caverna de Terra Boa, localizada no município de Rio Branco do Sul (PR). A única exceção é a camada superficial do sítio 11 , que apresentou maior acidez, que não pode ser atribuída a efeitos antrópicos atuais, visto que se trata do antigo nível de base do rio subterrâneo.

Os valores de $C$ orgânico são maiores nas camadas superficiais dos sedimentos, evidenciando duas hipóteses prováveis: contribuição dos organismos vivos que habitam a caverna e/ou contribuição de sedimentos mais recentes devido à erosão atual do ambiente de entorno. A segunda possibilidade pa- 
SIRTOLI, A.E. e LIMA, M.R. Caracterização preliminar de sedimentos...

rece não explicar totalmente este fato, pois são encontrados elevados níveis de $\mathrm{C}$ orgânico tanto em sítios no nível de base atual, como em leitos antigos ou áreas mais altas das cavernas. Bottrell (01), estudando a dinâmica de carbono marcado em cavernas calcárias, observou que esta acumulação nos sedimentos superficiais é relativamente constante no tempo, havendo a conseqüente mineralização durante a diagênese, não podendo ser atribuída simplesmente à ocorrência de práticas agrícolas no entorno com a aplicação de estercos e lodo urbano e seu posterior transporte para o interior da caverna. De fato, foi constatado nestas mesmas cavernas, abundante presença de organismos vivos, especialmente quirópteros, que são responsáveis por uma importante entrada de matéria orgânica, sendo que sobre o guano depositado no chão da caverna se desenvolve uma complexa biocenose (08). Como no ecossistema cavernícola há reduzida presença de microorganismos autotróficos, limitada à proximidade das aberturas naturais, a maior parte da matéria orgânica é alóctone, entrando no sistema através de correntes de água e ar, raízes de plantas e animais troglóxenos (08), embora possa existir uma população de microorganismos quimioautotróficos no interior da caverna.

Na caverna situada no sistema Pinheirinho, os teores de $\mathrm{C}$ orgânico foram menores que aqueles encontrados nas demais cavernas. Talvez este fato seja devido a esta caverna possuir um comprimento de
1290 m (06), sendo a maior da três estudadas, o que poderia conduzir à diluição da matéria orgânica trazida pelos animais troglóxenos ao longo deste percurso.

Os níveis elevados de $\mathrm{P}$ disponível, devem ser analisados com cuidado. O método de extração adotado pela maioria dos laboratórios de análises de solos no Paraná, extrator Mehlich-1 (09), é extremamente favorável a extração de fosfatos de $\mathrm{Ca}$, mesmo em formas não lábeis (10), que são formadas em condições de alcalinidade do solo. Outros métodos de extração, como o método da resina trocadora de ânions (12), talvez fossem mais indicados para este tipo de material. A grande quantidade de $\mathrm{P}$ disponível encontrada nos sedimentos das cavernas talvez pudesse ser associada ao acúmulo de matéria orgânica. Porém não se observa uma correlação em todas as situações. Como se observa no sítio 2 , o aumento da quantidade de $\mathrm{P}$ disponível não é atribuída ao aumento no teor de $\mathrm{C}$ orgânico. Sabe-se também que a rocha matriz destes sedimentos (calcário) é pobre em P. Porém, como a maioria dos materiais encontrados no interior das cavernas são oriundos de sedimentação, resta saber qual a origem destes sedimentos e composição química. Outra possibilidade relacionada ao alto teor de $\mathrm{P}$ disponível poderia ser o transporte de fertilizantes, orgânicos e inorgânicos, contendo o nutriente para o interior da caverna. Porém esta hipótese não poderia explicar os altos teores nas amostras coletadas nos sítios localizados em níveis de base pretéritos.

TABELA 1 - Características de fertilidade e granulométricas dos sedimentos situados no interior das cavernas estudadas.

\begin{tabular}{|c|c|c|c|c|c|c|c|c|c|c|c|c|c|c|c|c|}
\hline \multirow[t]{2}{*}{ SISTEMA } & \multirow[t]{2}{*}{ SÍTIO } & $\begin{array}{l}\text { PROFUN- } \\
\text { DIDADE }\end{array}$ & $\begin{array}{l}\mathrm{pH} \mathrm{em} \\
\mathrm{CaCl}_{2}\end{array}$ & $\mathrm{Al}^{+3}$ & $\mathrm{H}+\mathrm{Al}$ & $\mathrm{Ca}^{+2}$ & $\mathrm{Mg}^{+2}$ & $\mathrm{~K}^{+}$ & $\mathrm{CTC}_{1}$ & \multirow{2}{*}{$\begin{array}{l}\mathrm{V}^{2} \\
\%\end{array}$} & \multirow{2}{*}{$\begin{array}{c}\mathrm{P} \\
\text { disponível }\end{array}$} & \multirow{2}{*}{$\begin{array}{c}\mathrm{C} \\
\text { orgânico } \\
\mathrm{g} \mathrm{dm}^{-3}\end{array}$} & \multirow[t]{2}{*}{$\begin{array}{c}\text { AREA } \\
\text { GROSSA }\end{array}$} & \multirow[t]{2}{*}{$\begin{array}{c}\text { AREIA } \\
\text { FINA }\end{array}$} & \multirow[t]{2}{*}{ SILTE } & \multirow[t]{2}{*}{ ARGLA } \\
\hline & & \multicolumn{2}{|l|}{$\mathrm{cm}$} & \multicolumn{6}{|c|}{$\mathrm{cmol}_{\mathrm{c}} \mathrm{dm}^{-3}$} & & & & & & & \\
\hline \multirow{8}{*}{ CACHMBA } & \multirow[t]{2}{*}{01} & $0-20$ & 6,8 & 0,0 & 1,8 & 10,6 & 2,0 & 0,21 & 14,6 & 88 & 80 & 4,8 & 26 & 4 & 40 & 30 \\
\hline & & $40-60$ & 7,2 & 0,0 & 1,6 & 7,2 & 2,4 & 0,20 & 11,4 & 86 & 25 & 2,1 & 28 & 8 & 14 & 50 \\
\hline & \multirow[t]{2}{*}{02} & $0-20$ & 6,1 & 0,0 & 2,7 & 10,4 & 2,1 & 0,07 & 15,3 & 82 & 7 & 19,0 & 0 & 8 & 46 & 46 \\
\hline & & $40-60$ & 7,0 & 0,0 & 1,9 & 10,7 & 1,9 & 0,12 & 14,6 & 87 & 49 & 4,8 & 18 & 8 & 32 & 42 \\
\hline & 03 & $0-20$ & 7,2 & 0,0 & 1,6 & 9,6 & 1,9 & 0,15 & 13,3 & 88 & 350 & 4,8 & 16 & 24 & 34 & 26 \\
\hline & \multirow[t]{2}{*}{04} & $0-20$ & 7,3 & 0,0 & 1,7 & 12,4 & 1,9 & 0,15 & 16,2 & 89 & 28 & 8,2 & 4 & 26 & 50 & 20 \\
\hline & & $40-60$ & 7,4 & 0,0 & 1,6 & 9,2 & 1,5 & 0,07 & 12,4 & 87 & 40 & 6,9 & 30 & 18 & 26 & 26 \\
\hline & 05 & $0-20$ & 7,2 & 0,0 & 1,9 & 10,5 & 2,2 & 0,15 & 14,8 & 87 & 350 & 15,6 & 4 & 18 & 54 & 24 \\
\hline \multirow{5}{*}{ BROMADO } & 06 & $0-20$ & 7,2 & 0,0 & 1,8 & 15,5 & 0,8 & 0,13 & 18,2 & 90 & 145 & 8,2 & 4 & 6 & 48 & 42 \\
\hline & 07 & $0-20$ & 6,4 & 0,0 & 1,5 & 10,0 & 1,6 & 0,43 & 13,5 & 89 & 190 & 3,4 & 10 & 14 & 58 & 18 \\
\hline & & $40-60$ & 7,2 & 0,0 & 1,5 & 10,5 & 2,0 & 0,45 & 14,5 & 89 & 46 & 4,8 & 2 & 16 & 50 & 32 \\
\hline & 08 & $0-20$ & 7,0 & 0,0 & 1,5 & 13,2 & 2,1 & 0,08 & 16,9 & 91 & 210 & 2,1 & 2 & 4 & 38 & 56 \\
\hline & 09 & $0-20$ & 7,2 & 0,0 & 1,6 & 7,1 & 1,7 & 0,06 & 10,5 & 85 & 836 & 2,1 & 24 & 6 & 36 & 34 \\
\hline \multirow{5}{*}{ PNHERNHO } & 10 & $0-20$ & 6,6 & 0,0 & 2,5 & 11,6 & 2,0 & 0,39 & 16,5 & 85 & 704 & 2,1 & 2 & 20 & 58 & 20 \\
\hline & 11 & $0-20$ & 4,2 & 2,0 & 7,2 & 3,0 & 2,3 & 0,11 & 12,6 & 43 & 26 & 1,1 & 2 & 26 & 52 & 20 \\
\hline & & $100-120$ & 5,9 & 0,0 & 3,2 & 12,0 & 2,7 & 0,16 & 18,1 & 82 & 46 & 1,1 & 0 & 8 & 48 & 44 \\
\hline & & $180-200$ & 6,3 & 0,0 & 2,0 & 8,5 & 2,8 & 0,12 & 13,4 & 85 & 61 & 1,1 & 2 & 22 & 52 & 24 \\
\hline & 12 & $0-20$ & 6,4 & 0,0 & 2,2 & 9,5 & 1,6 & 0,17 & 13,5 & 84 & 56 & 6,9 & 14 & 30 & 42 & 14 \\
\hline
\end{tabular}

${ }^{1}$ Capacidade de troca de cátions.

${ }^{2}$ Saturação por bases. 
SIRTOLI, A.E. e LIMA, M.R. Caracterização preliminar de sedimentos...

À exceção dos sítios 7 e 10, os teores de $\mathrm{K}$ trocável nas três cavernas levantadas situaram-se entre valores médios a baixos. Isto provavelmente se deve ao fato do $\mathrm{K}$ ser um elemento móvel no solo, facilmente lixiviável, devido a presença de alta umidade nos sedimentos das cavernas.

A CTC e o valor $V$ nos sedimentos estudados são considerados altos, à exceção da saturação por bases da camada superficial do sítio 11, que foi considerada baixa, fato esperado tendo em vista possuir $\mathrm{pH}$ mais ácido. Os valores altos de $\mathrm{V}$ são coerentes com o pH encontrado nos sedimentos, pois na faixa de baixa acidez ou subalcalinidade a acidez trocável tende a ser neutralizada e os cátions básicos $\left(\mathrm{Ca}^{+2}\right.$, $\mathrm{Mg}^{+2}, \mathrm{~K}^{+}$) ocupam a maior parte da CTC. A análise da atividade da fração argila permite identificar que nos sedimentos das cavernas dos sistemas Cachimba e Bromados devem predominar minerais com baixa CTC, enquanto que na fração argila dos sedimentos da caverna do sistema Pinheirinho devem predomi- nar minerais com elevada CTC, como os argilominerais tipo 2:1.

Em relação à composição granulométrica, pode-se observar que há uma tendência dos sedimentos possuírem textura argilosa ou média, o que vem ao encontro dos resultados encontrados por Basso (02) na caverna Terra Boa.

Aparentemente, os elevados teores de areia grossa de amostras retiradas nos sítios 1, 4 e 12, poderiam ser indícios de degradação ambiental do entorno da caverna. Segundo Costa (04) e Chaimovicz (03), o solo cavernícola, por suas características, deveria apresentar alta proporção de fração argila, o que se evidencia em algumas situações de subsuperfície, especialmente no nível de base atual. No entanto, as amostras retiradas nos sítios 2 , 3 e 9 também apresentam elevados teores de areia grossa, o que não corrobora a suposição da associação com a degradação antrópica do ambiente cárstico externo à caverna.

\section{BIBLIOGRAFIA}

1 BOTTRELL, SH. Organic carbon concentration profiles in recent cave sediments: records of agricultural pollution or diagenesis? Environmental Pollution, v. 91, n. 3, p. 325-332, 1996.

2 BASSO, RL. Aspectos geomorfológicos e análises físico-químicas e biológicas do solo de cavernas da Região Metropolitana da Curitiba, Paraná, Brasil. Curitiba, 1990. 81 f. Monografia (Bacharel em Biologia) - Departamento de Ciências Biológicas, Centro de Ciências Biológicas e da Saúde, Pontifícia Universidade Católica do Paraná.

3 CHAIMOWICZ, F. Bioespeleologia: o estudo da biologia das cavernas. São Paulo: SP Editorial, 1986. 52p

4 COSTA, JB. Caracterização e constituição do solo. 2 ed. Lisboa: Calouste Gulbenkian, 1979. 527p.

5 EMBRAPA. Centro Nacional de Pesquisa de Solos. Manual de métodos de análise de solos. 2 ed. Rio de Janeiro, 1997. 212 p. (EMBRAPA-CNPS. Documentos, 01).

6 GENTHNER, C.; SILVA-DA-ROCHA, LF.; ZAKRZEWSKI, DP.; TALAMINI NETO, E. Noções preliminares sobre o sistema cárstico da gruta Pinheirinho. In: GRUPO DE ESTUDOS ESPELEOLÓGICOS DO PARANÁ-AÇUNGUI. Cavernas do Paraná: dez anos de espeleologia GEEP-Açungui. Curitiba, 1996.

7 HARWICK, P.; GUNN, J. The conservation of Britain's limestone cave resource. Environmental Geology, New York, v. 28, p. $121-127$, Oct. 1996.

8 INSTITUTO DE ESTUDOS AMBIENTAIS MATER NATURA. Relatório técnico final. Projeto: Observações ecológicas em cavernas da região metropolitana de Curitiba - análise do sistema cárstico. Curitiba, 1997.173 p.

9 PAVAN, MA.; BLOCH, MF.; ZEMPULSKI, HC.; MIYAZAWA, M.; ZOCOLER, DC. Manual de análise química de solo e controle de qualidade. Londrina: IAPAR, 1992. 40 p. (IAPAR. Circular, 76).

10 RAIJ, B. van; QUAGGIO, JA. Métodos de análise de solo para fins de fertilidade. Campinas: Instituto Agronômico, 1983 (IAC. Boletim técnico, 81).

11 RAIJ, B. van. Fertilidade do solo e adubação. São Paulo; Piracicaba: Ceres, Potafos, 1991. 343 p.

12 RAIJ, B. van; QUAGGIO, JA.; SILVA, NM. Extraction of phosphorus, potassium, calcium and magnesium from soils by an ion exchange resin procedure. Communications in Soil Science and Plant Analysis, New York, v. 17, p. 547-566, 1986.

13 SESSEGOLO, GC.; ZAKRZEWSKI, DP.; THEULEN, U.; SILVA-DA-ROCHA, LF. A degradação ambiental em cavernas da região metropolitana de Curitiba-PR. In: GRUPO DE ESTUDOS ESPELEOLÓGICOS DO PARANÁ-AÇUNGUI. Cavernas do Paraná: dez anos de espeleologia GEEP-Açungui. Curitiba, 1996. 